\title{
Buruli ulcer in Nigeria: results of a pilot case study in three rural districts
}

Kingsley N. Ukwaja ${ }^{1 *}$ (D, Anthony O. Meka ${ }^{2}$, Alphonsus Chukwuka ${ }^{3}$, Kingsley B. Asiedu', Kristina L. Huber ${ }^{5}$, Miriam Eddyani ${ }^{6}$, Joseph N. Chukwu², Moses C. Anyim², Charles C. Nwafor ${ }^{2}$, Daniel C. Oshi ${ }^{2}$, Nelson O. Madichie ${ }^{2}$, Ngozi Ekeke ${ }^{2}$, Martin Njoku ${ }^{3}$ and Kentigern Ntana ${ }^{3}$

\begin{abstract}
Background: Buruli ulcer (BU), also known as Mycobacterium ulcerans disease, is the third most common mycobacterial disease worldwide. Although BU disease has been diagnosed among Nigerians in neighbouring West African countries, data on the burden of the disease in Nigeria itself are scanty. This study aimed to assess the magnitude and epidemiology of BU in the South South region of Nigeria.
\end{abstract}

Methods: We conducted a cross-sectional survey in the Ogoja territory (comprising 31 communities). We undertook sensitisation programmes centred on BU in 10 of the communities. Participants were asked to identify community members with long-standing ulcers, who were then invited for evaluation. We also contacted traditional healers to refer their clients who had non-healing ulcers. All suspected cases had a full clinical evaluation and laboratory testing. Confirmed cases were given treatment in a referral hospital in the territory.

Results: We diagnosed 41 clinical BU cases; 36 (87.8\%) of which were confirmed by quantitative polymerase chain reaction (qPCR). These 36 PCR-confirmed cases were diagnosed in a total population of 192,169 inhabitants. Therefore, the estimated crude prevalence of BU was 18.7 per 100,000 population, varying from 6.0 to 41.4 per 100,000 in the districts surveyed. The majority (66.7 \%) of the cases were females. About $92 \%$ of the BU lesions were located on the patients' extremities. No differences were observed between the sexes in terms of the location of the lesions. The age of the patients ranged from four to 60 years, with a median age of 17 years. All 35 (100\%) patients who consented to treatment completed chemotherapy as prescribed. Of the treated cases, 29 (82.9\%) needed and received surgery. All cases healed, but 29 (82.9\%) had some limitations in movement. Healing with limitations in movement occurred in 18/19 (94.7\%) and 8/10 (80.0\%) of patients with lesions $>15 \mathrm{~cm}$ (Category III) and $6-15 \mathrm{~cm}$ in diameter (Category II), respectively. The median duration of treatment was 130 (87-164) days for children and 98 (56-134) days for adults $(p=0.15)$.

Conclusions: In Nigeria, BU is endemic but its severity is underestimated-at least in the study setting. There is a need to identify and map BU endemic regions in Nigeria. A comprehensive BU control programme is also urgently needed.

Keywords: Buruli ulcer, Mycobacterium ulcerans, Epidemiology, Case finding, Endemicity, Ogoja, Cross River State, Nigeria

\footnotetext{
* Correspondence: ukwajakingsley@yahoo.co.uk

${ }^{1}$ Department of Medicine, Federal Teaching Hospital , FMC Rd, Abakaliki,

Ebonyi State, Nigeria

Full list of author information is available at the end of the article
} 


\section{Multilingual abstracts}

Please see Additional file 1 for translations of the abstract into the six official working languages of the United Nations.

\section{Background}

Buruli ulcer (BU), a neglected tropical disease, is caused by Mycobacterium ulcerans. Globally, it is the third most common mycobacteriosis after tuberculosis and leprosy [1]. The infection leads to the destruction of skin and soft tissue, presenting as large ulcers usually on the limbs. Patients who are not treated early can suffer permanent disfigurement and functional disability $[1,2]$. Buruli ulcer has been reported in 33 countries in Africa, the Americas, Asia and the Western Pacific. Most cases occur in tropical and subtropical regions, except for in Australia, China and Japan [1, 2]. In West and Central Africa, Benin, Cameroon, Côte d'Ivoire, Democratic Republic of Congo (DRC) and Ghana have reported the majority of cases [3-7].

The exact mode of transmission of $M$. ulcerans is still unknown [1,2]. Epidemiological studies have shown that $\mathrm{BU}$ is commonly found in populations living near rivers, swamps and wetlands $[1,2,8]$. In several instances, local environmental events, such as deforestation, flooding and building of dams, or agricultural activities such as irrigation, have been associated with the emergence of $\mathrm{BU}[1,8]$. The risk factors for BU that have been repeatedly identified include proximity to stagnant or slow-flowing bodies of water, poor wound care and not wearing protective clothing (e.g., long-sleeved shirts or shoes) $[1,8]$. The probable role of aquatic insects as reservoirs and vectors of $M$. ulcerans has been proposed, but is still being debated [1, 8, 9].

Although Nigeria is surrounded by countries with a high endemicity of BU disease, only few cases have been reported to date. In 1967, four BU cases were first reported from Benue, Nigeria [10]. This was followed by 24 cases reported from Ibadan, in Oyo state, in 1976 [11]. Based on unofficial reports from some states in Southern Nigeria, the World Health Organization (WHO) carried out a rapid assessment in 2006, finding 14 cases with clinically-suspected BU [12]. In addition, a recent report mentioned that nine $M$. ulcerans strains were isolated from patients living in Oyo, Anambra, Cross River, Enugu, Ebonyi and Ogun states, during 2006 and 2012 [13]. Therefore, to date, 51 cases of BU have been reported from Nigeria. However, given the high number of BU patients from Nigeria being treated in neighbouring countries, such as Benin and Cameroon $[14,15]$, there seems to be a discrepancy, likely due to underreporting and inadequate public health structures to diagnose and treat the disease in Nigeria.

Furthermore, the WHO reports that there is evidence that BU disease is gradually increasing in incidence and that its geographic range is also increasing [16]. Given that the disease can suddenly appear in a new area which has previously been disease-free, especially in poor rural communities in Sub-Saharan Africa and other developing countries, basic data to help plan effective BU control activities are urgently needed, but lacking $[1,16]$. The main objective of this pilot project was to ascertain the extent of BU disease in rural communities of Nigeria and provide an evidence base for a systematic national response. The specific objectives were to assess the minimum prevalence of $\mathrm{BU}$ and to determine the epidemiologic characteristics of BU in Ogoja territory, the target endemic region of the project.

\section{Methods}

\section{Study area}

The study was conducted in the Ogoja territory of Cross River State, Nigeria (see Fig. 1). The territory is a tropical rainforest belt surrounded by rivers and swamps. Cross River State derives its name from the Cross River, which transcends almost the entire length of the state and empties into the Atlantic (covering an area of $39,000 \mathrm{~km}^{2}$ ). The majority of the state's inhabitants utilise the Cross River as source of drinking water, and most engage in farming of rice, yam and cassava. Three Local Government Areas (LGAs), i.e. Ogoja, Yala and Bekwarra, which previously constituted Ogoja territory, were selected as the study sites due to prior notification of BU in the region [12]. There are 31 communities in the three LGAs. Ten communities, which constitute about one third of the population of the three LGAs, were selected for the survey through simple random sampling, covering an estimated population of 192,169 people [17]. The study communities have 12 primary health centres and four secondary health care facilities, including the St Benedict Tuberculosis and Leprosy Rehabilitation Hospital Ogoja (SBHO), which is a private not-for-profit (faith-based) hospital that serves as a referral centre for tuberculosis and leprosy control in the whole of Ogoja.

\section{Study design}

This was a community-based cross-sectional survey carried out between May 2012 and April 2013. It was conducted in two phases: a preparatory, and a casefinding and management phase. During the first three weeks of the project (May 2012), advocacy visits were held with the community leaders and health authorities, and their approval for the study was obtained. Project staff members in the facilities were trained on identification of BU suspects, sample collection, preparation and transportation. General health workers and doctors working in the peripheral health facilities in the three study LGAs were trained on BU symptoms recognition and appropriate referral. 


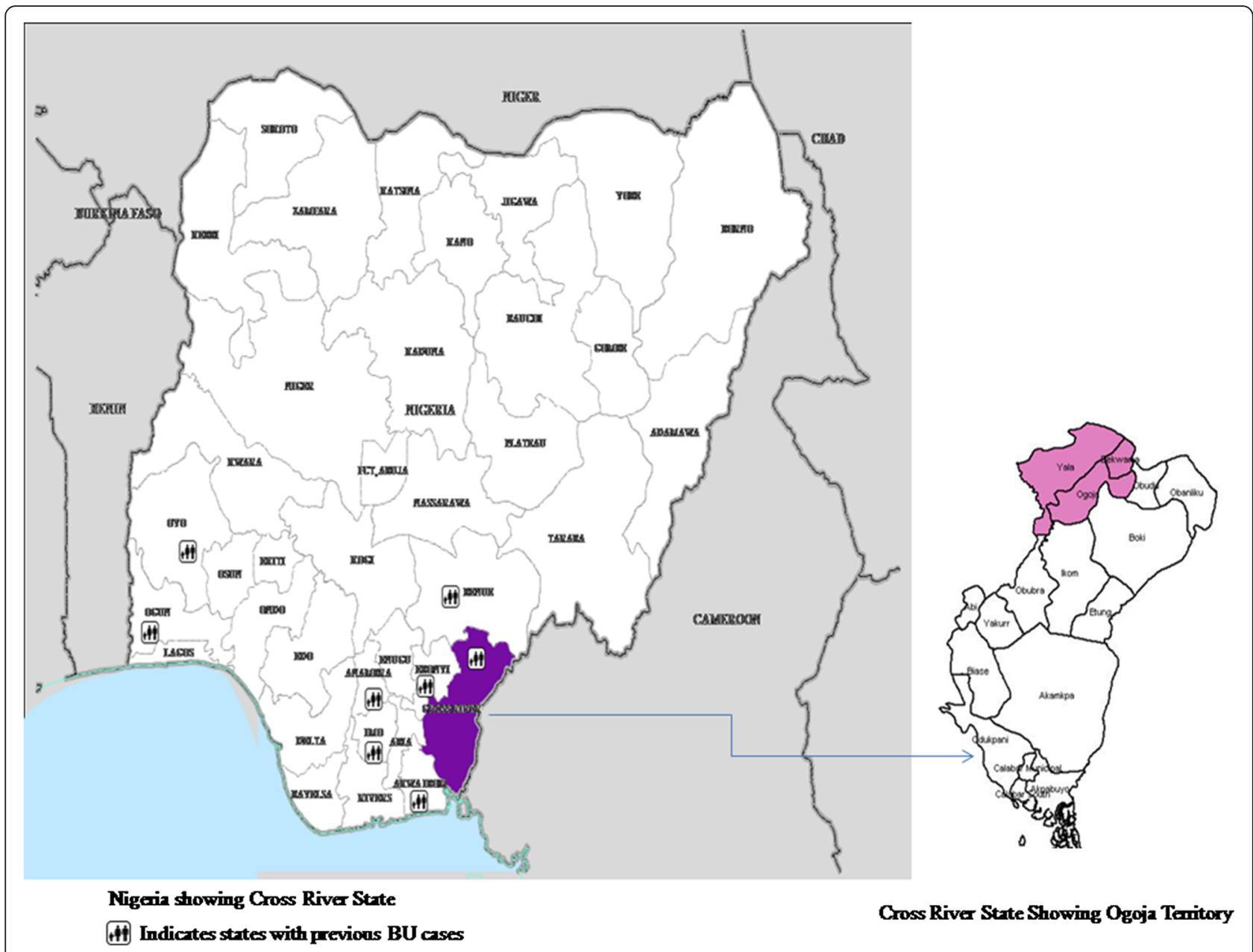

Fig. 1 A map of Nigeria showing states where BU has been previously reported, highlighting the Cross River State and the three pilot project LGAs

The case-finding and management phase involved intensive advocacy, communication and social mobilisation. Due to logistical problems, it was not possible to undertake a door-to-door survey. However, outreach/ sensitisation activities took the form of village hall/ square meetings with the whole community. Community leaders were informed of the intention to hold outreach programmes in their community, a date was chosen for the event, community members were instructed to gather at the village hall/square, and finally the community sensitisation programme was undertaken. This was done monthly in all study communities. During the sensitisation activities, the communities were informed about the free BU treatment opportunities at the SBHO. Case finding formed part of the advocacy, communication and social mobilisation activities. Screening for BU disease was done onsite and persons clinically suspected were referred to the SBHO for evaluation, free laboratory investigation and treatment. In addition, traditional healers in each community were interviewed about managing individuals with chronic ulcers that have failed to heal. They were also trained to refer such cases for evaluation at the SBHO. Individuals suspected to have $\mathrm{BU}$ disease were interviewed about whether they knew other people who had similar lesions. Such cases were identified, evaluated and referred to the SBHO. Patients suspected of having $\mathrm{BU}$ disease that were referred to or presented at the SBHO had their diagnoses clinically validated by trained physicians to ensure that they were consistent with the WHO clinical case definitions $[18,19]$, and subsequently had other management strategies initiated.

\section{Laboratory confirmation}

Laboratory confirmation of clinical BU disease cases involved taking swabs from ulcerative lesions and fine needle aspirates from pre-ulcerative lesions, followed by laboratory testing (microscopy and/or molecular biology) using the WHO guidelines [18]. Samples were also sent to the Institute of Tropical Medicine Antwerp and the 
Division of Infectious Diseases and Tropical Medicine, Medical Centre of The University of Munich, where quantitative polymerase chain reaction (qPCR) was performed for the detection of $M$. ulcerans.

\section{Clinical care and management}

Case management was carried out according to the WHO recommendations [19]. Each patient received an eight-week drug treatment (chemotherapy) using the standard regimen of rifampicin and streptomycin. Wound dressing for ulcerated cases was an important component of clinical management. For each patient, this process continued until the wound had either healed completely or adequately granulated for skin grafting. Surgery was part of the ulcer management, as some of the cases required surgical interventions such as debridement, skin grafting and/or amputation. Physiotherapy was provided to those who had contractures and limitation in movement.

\section{Statistical analysis}

The data were recorded on a standardised BU report form, double-entered into a Microsoft Excel (Microsoft Office Inc for Windows, USA) database and analysed using Epi Info version 3.4.1 (Centers for Diseases Control and Prevention, Atlanta, GA, USA). Continuous variables were summarised as medians (interquartile ranges), and categorical variables as counts and percentages. The Fisher's exact or chi-square test was used to compare categorical proportions, and the Student's $t$-test or Mann-Whitney $U$ test was used in the case of continuous variables. The significance level was set at $5 \%$.

\section{Ethics statement}

Ethical approval for this study was obtained from the Ethical Advisory Board of the German Tuberculosis and Leprosy Relief Association. Permission was also obtained from the management of the SBHO. All patients or their legal guardians (for minors) gave written informed consent for all diagnostic and treatment purposes, as well as for the publication of clinical photographs. All BU cases part of the study were treated at no financial costs to them.

\section{Results}

\section{Laboratory confirmation of cases}

In the one-year period of the project, 41 patients with clinical BU lesions at different stages of development were identified. Of these, 36 (87.8 \%) were confirmed by qPCR. Of the cases not confirmed in the laboratory, four $(9.8 \%)$ tested negative by qPCR and one $(2.4 \%)$ didn't have samples collected. In addition, $45.2 \%(14 / 31)$ of the clinically confirmed BU cases who had a smear examination also tested positive using Ziehl-Neelsen staining-all of whom were also positive for BU using the qPCR method. We restricted our subsequent report to the 36 cases who were positive for BU by qPCR.

\section{Clinical presentation}

All 36 (100 \%) patients were newly diagnosed BU disease cases. About $88.9 \%$ of the lesions were at the ulcerative stage (see Fig. 2) and $11.1 \%$ were at the pre-ulcerative stage. Lesions occurring at both the ulcerative and preulcerative stages were seen in $2.8 \%$ of the patients. Table 1 shows the clinico-epidemiological features of the cases. The locations of the lesions were variable: 19

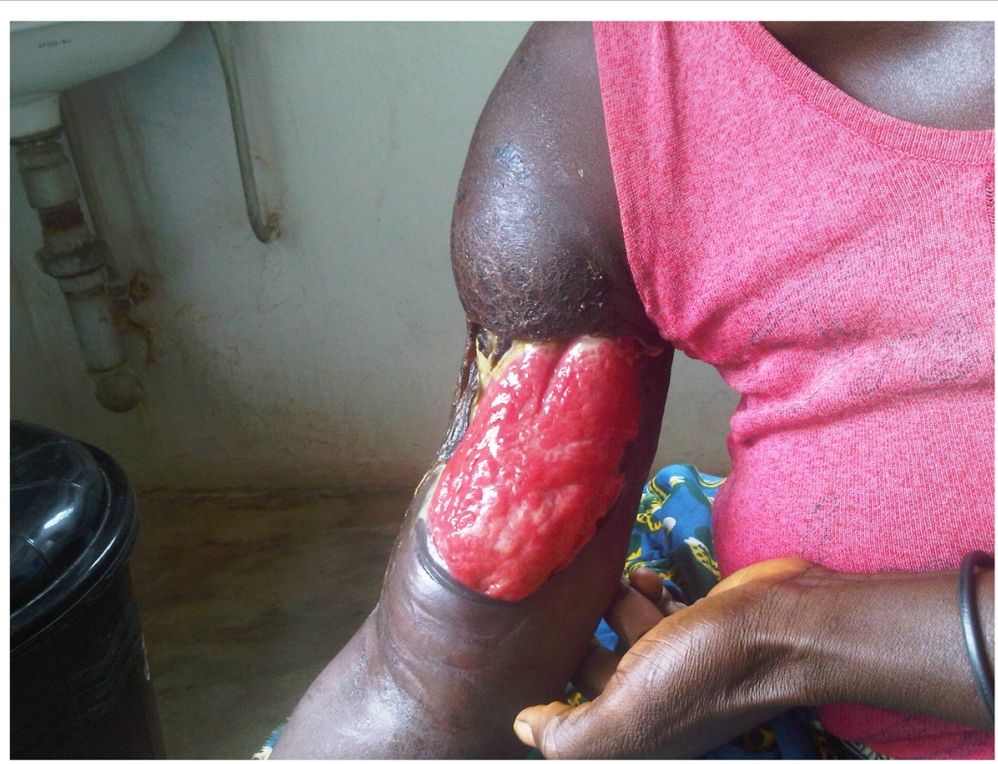

Fig. 2 A typical Buruli ulcer on the upper limb of an adult male identified during the study 
Table 1 Clinico-epidemiological features of Buruli ulcer cases in Ogoja territory, May 2012 to April 2013

\begin{tabular}{lll}
\hline Characteristics & & Buruli ulcer case $\mathrm{n}(\%)$ \\
\hline Sex & Female & $24(66.7)$ \\
& Male & $12(33.3)$ \\
Age group & $\leq 15$ years & $15(41.7)$ \\
& $>15$ years & $21(58.3)$ \\
Classification of cases & New case & $36(100)$ \\
& Recurrent & $0(0)$ \\
Clinical form & Plaque & $4(11.1)$ \\
& Ulcer & $31(86.1)$ \\
Site of lesion & Ulcer and oedema & $1(2.8)$ \\
& Upper limb & $14(38.9)$ \\
& Lower limb & $19(52.8)$ \\
Size of lesion & Trunk & $3(8.3)$ \\
& Category I & $6(16.7)$ \\
& Category II & $11(30.6)$ \\
& Category III & $19(52.8)$ \\
Limitation of movement? & Yes & $24(66.7)$ \\
& No & $12(33.3)$ \\
\hline
\end{tabular}

(52.8\%) had lesions on the lower limb, 14 (38.9\%) had lesions on the upper limb and three (8.3\%) had lesions on the trunk. More than half $(52.8 \% ; 19)$ of the patients presented advanced ulcer lesions that were more than $15 \mathrm{~cm}$ in diameter (Category III lesions) (see Fig. 3), the majority of which occurred in females (58.3 \% vs. $41.7 \%$; $p=0.35)$; while $11(30.6 \%)$ had lesions that were 6$15 \mathrm{~cm}$ in diameter (Category II lesions). In addition, 24 (67\%) had functional limitations in movement, with these limitations most significantly occurring (58.3\%-14 patients) in patients with lesions on the lower limb.

\section{Epidemiological features}

Using the identified active lesions (pre-ulcerative or ulcerative) and the populations of the study communities, we estimated that the crude minimum prevalence rate was 18.7 per 100, 000. Table 2 shows the minimum prevalence and distribution of BU disease cases according to the size of the population where the survey was conducted. Ogoja LGA had the highest prevalence rate of 41.4 per 100,000 population. Of the PCR-confirmed BU cases, 24 (66.7 \%) were female and 12 (33.3\%) were male. The ages of the patients ranged from four to 60 years (median: 17 years, mode: 20 years). Overall, 15 (41.7\%) were children ( $\leq 15$ years). No statistical difference was observed in terms of sex among patients who were children $(\leq 15$ years $)$ compared with adults $>15$ years old (Fisher's exact test: $p=0.14$ ) (see Table 1).
The distribution of lesions on the limbs and trunk did not differ among the sexes $\left(\chi^{2}=1.4 ; p=0.24\right)$. Among females, lesions occurred equally $(45.8 \%)$ on the upper limb and the lower limb, while the remaining $8.4 \%$ occurred on the trunk. Among males, $66.7 \%$ of the lesions occurred on the lower limb, $25.0 \%$ on the upper limb and $8.3 \%$ on the trunk. The distribution of the lesions also did not differ by age group (Fisher's exact test: $p=$ 0.455). Among children, $53.3 \%$ of the lesions occurred on the lower limb, $40 \%$ on the upper limb and $6.7 \%$ on the trunk. Among adults, 52.4\% of lesions occurred on the lower limb, $38.1 \%$ on the upper limb and $9.5 \%$ at the trunk.

\section{Case management}

Of the cases diagnosed, 35 were treated with antibiotics (oral rifampicin and streptomycin injection) for eight weeks, while one patient declined treatment. Of the treated cases, 29 (82.9\%) needed and received surgery. All 35 treated cases completed treatment and were discharged. Figure 4 shows the progression of one case's healing process. Of the total number of cases, 29 $(82.9 \%)$ healed with some limitations in movement, while six $(17.1 \%)$ cases healed with no limitation in movement. Healing with limitations in movement occurred in 18/19 (94.7\%), 8/10 (80.0\%) and $3 / 6$ $(50 \%)$ of patients with Category III $(>15 \mathrm{~cm})$, Category II $(6-15 \mathrm{~cm})$ and Category I $(\leq 5 \mathrm{~cm})$ lesions, respectively (see Fig. 5). All the cases that healed with limitations in joint movement received physiotherapy, with some improvements observed. Overall, the treatment duration (including hospitalisation/dressings) ranged from 56 to 244 (median of 135) days. Patients with Category III, II and I lesions had median durations of treatment of 120, 116 and 83 , days, respectively $(p=0.37)$. The median duration of treatment was 130 (87-164) days for children and 98 (56-134) days for adults $(p=0.15)$.

\section{Discussion}

The overall crude prevalence rate of $\mathrm{BU}$ of 18.7 per 100,000 in the study communities (and the much higher rate of 41.4 per 100,000 in Ogoja) indicates that BU is prevalent in the region, and particularly in Ogoja territory. Before this pilot project, a cumulative total of 51 BU cases have been reported from Nigeria [15]—only three of these were from Ogoja LGA [12]. This suggests that in endemic communities in Nigeria, the number of BU cases is grossly underestimated. Therefore, there is an urgent need to step up BU case finding in communities where standard reporting has previously identified at least one $\mathrm{BU}$ case. The crude prevalence rate of $\mathrm{BU}$ found in this study is comparable to the 21 and 30 per 100,000 cases found in nationwide surveys in Ghana and Cote d'Ivoire, respectively [6, 7]. However, our finding 


\section{Male $\quad$ Female}

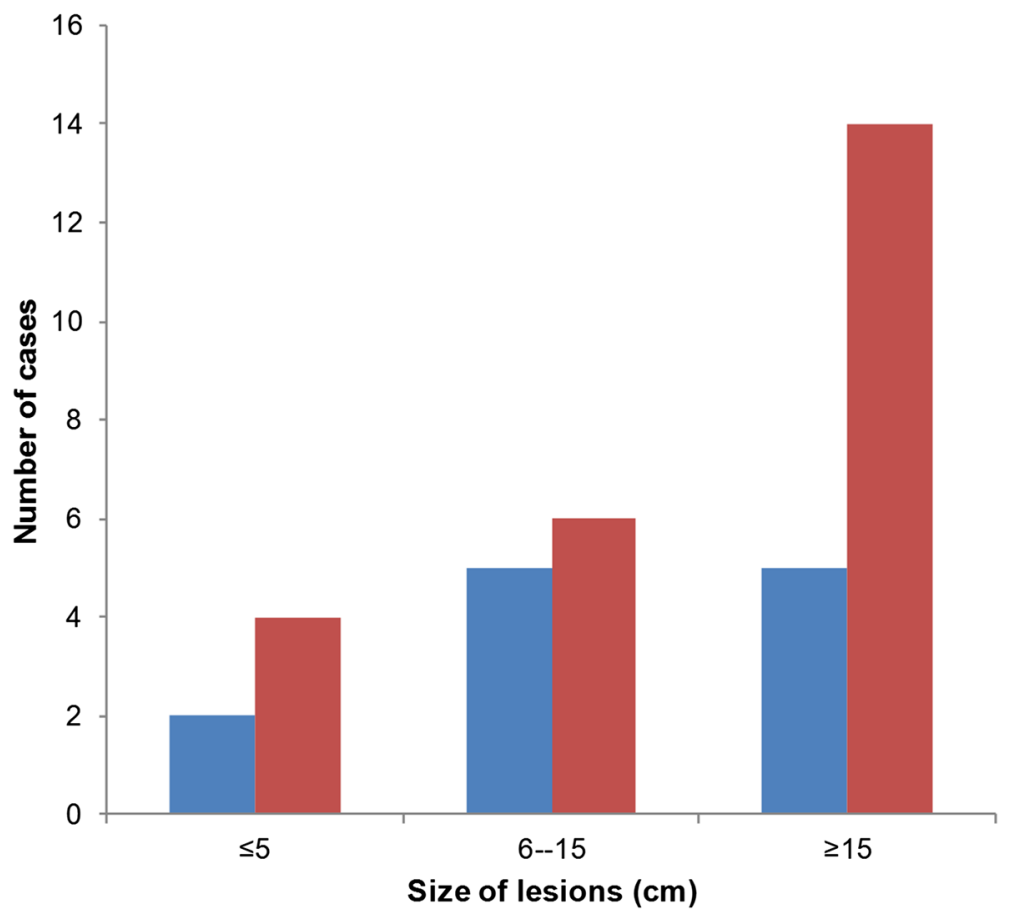

Fig. 3 Distribution and sizes of Buruli ulcer lesions, according to patients' sex

was lower than 184, 205 and 330 per 100,000 population reported in a district in Benin, Cameroon and DRC, respectively $[5,14,20,21]$. In our current effort, we have probably missed several prevalent BU cases that would have been identified if more active case-finding activities were implemented. Although BU is a notifiable disease in Nigeria, there have not been any deliberate efforts to engage the affected communities to identify and treat the disease. One of the commonly held notions by heath policymakers in Nigeria is that BU disease is not endemic in the country. The findings of this study disagree with this belief and justify the need for the institution of deliberate efforts to identify and control BU.

The median age of cases in this study was comparable to patients aged 15.5 years, as reported from Cameroon [20]. It was lower than the median age observed in

Table 2 Prevalence of Buruli ulcer in the Ogoja territory, May 2012 to April 2013

\begin{tabular}{llllc}
\hline LGA & $\begin{array}{l}\text { Number of } \\
\text { communities }\end{array}$ & Population & $\begin{array}{l}\text { Number of } \\
\text { BU cases }\end{array}$ & $\begin{array}{l}\text { Crude prevalence } \\
\text { per }\end{array}$ \\
\hline Bekwarra & 1 & 41,623 & 3 & 7.2 \\
Ogoja & 7 & 67,614 & 28 & 41.4 \\
Yala & 2 & 82,932 & 5 & 6.0 \\
Total & 10 & 192,169 & 36 & 18.7 \\
population & & & & \\
\hline
\end{tabular}

Ghana (25 years) and DRC (27 years), but higher than the median age of 12 years reported in Benin $[5,6,22]$. Overall, our study agrees with previous studies that found that the highest rate of the disease is observed among children $<15$ years of age in disease-endemic communities [5-7, 21, 22]. In addition, we found that $>60 \%$ of cases occurred in females. Previous studies didn't observe any statistical differences in the occurrence of cases among the sexes $[3,6,14,22]$. This difference may be attributable to a higher proportion of females participating in sensitisation and project activities in the communities, and therefore those with lesions had a higher likelihood to be screened and assessed, as census data suggest no female sex dominance in the population [17].

About $92 \%$ of the BU cases had lesions on their extremities in our study. This is consistent with observations in Ghana, Côte d'Ivoire and DRC, which found that more than $90 \%$ of the lesions occur on the limbs [5-7]. Females had an equal number of lesions on the upper and lower limbs, while males predominantly had lesions on the lower limbs. The reason/s for this difference is not clear, but this finding is consistent with the findings of Barker [23]. However, recent surveys have observed no differences among the sexes in the distribution of lesions $[3,6,14]$.

The most common clinical presentation was an ulcerative lesion, which occurred in almost $90 \%$ of the cases. 


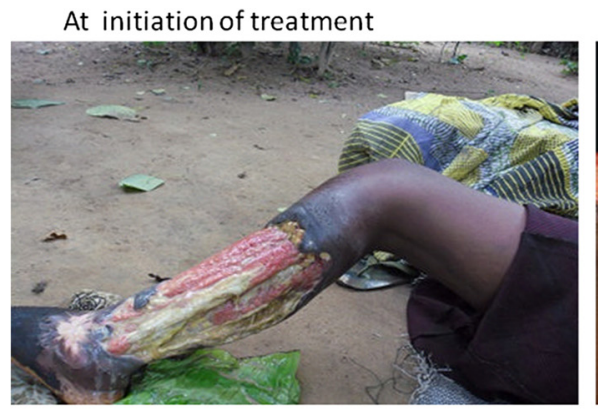

After eight weeks of treatment

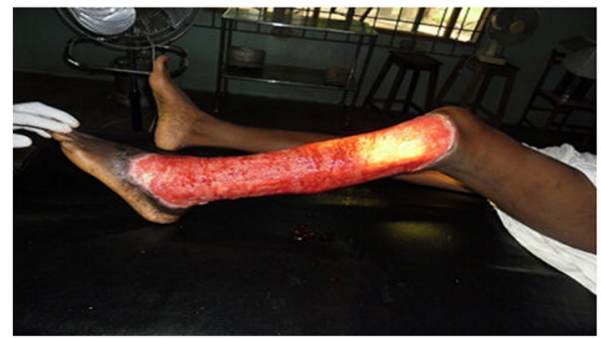

After four weeks of treatment

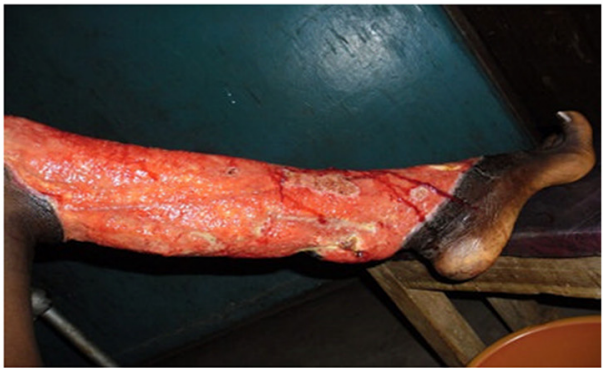

After twelve weeks of treatment

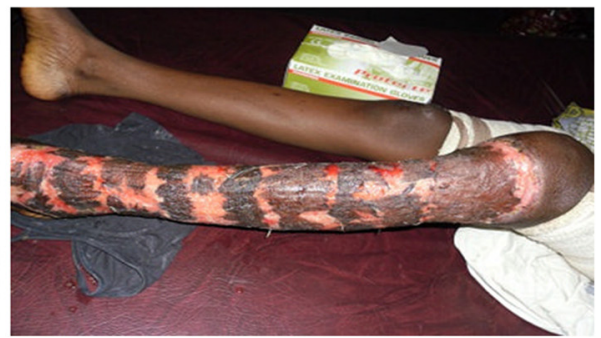

Fig. 4 Progressive healing of a Buruli ulcer patient who was given treatment

This agrees with studies conducted in the DRC, Cote d'Ivoire and Cameroon [4, 5, 7, 14]; while the proportion of patients with ulcerative lesions was lower in previous surveys undertaken in Ghana and Benin, which recorded proportions of ulcerative lesions ranging from $48 \%$ to $58 \%[3,6,21]$. Furthermore, two-thirds $(67 \%)$ of the cases had functional limitations of joint movement at presentation. This contrasts with previous findings which show that this occurs in less than one third of cases in other African endemic regions [5, 20]. This may be because more than half of the BU cases in this study had Category III lesions and were prone to complications. Moreover, late presentation due to a lack of BU control activities in Nigeria may have contributed to BU-related complications, resulting in a high proportion of cases with limitations of joint movement. Previous reviews have suggested an equal distribution of Category I, II, and III lesions [1]. The higher proportion of Category

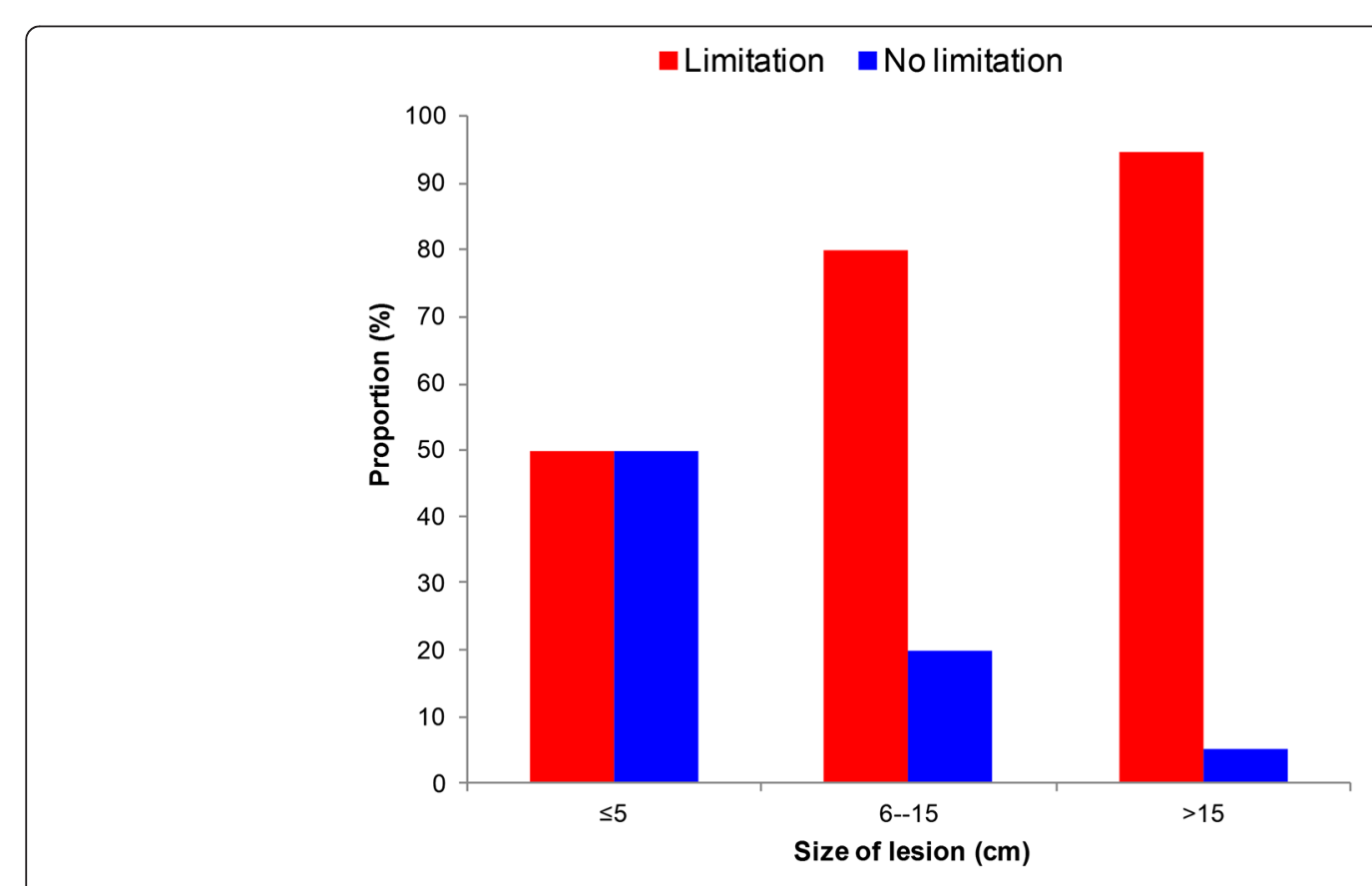

Fig. 5 Proportion of healed Buruli ulcer cases with or without limitation in movement, according to size of the lesion 
III lesions observed in this study may partly be explained by the study design, which focused on finding individuals with chronic ulcers in the community.

In addition, the identification of patients with more advanced ulcerative lesions may have allowed easier quality sample collection, which contributed to the high rate of laboratory confirmation of clinical cases. Most studies in other endemic regions in Africa had rates of laboratory-confirmed BU cases ranging from $30 \%$ to $74 \%$ [24-27]. Thus, the high rate of laboratory-confirmed cases in this survey may reflect a higher endemicity of active BU cases in the study communities than was previously observed. Moreover, given that only relatively few cases had Category I and II lesions, future surveys should target households, in order to increase the detection, diagnosis and treatment of individuals with early BU lesions in the community. Also, project staff and general health workers should be trained to identify early lesions of $\mathrm{BU}$ (i.e. oedema, nodules, etc.).

All treated BU cases completed chemotherapy and none were lost to follow-up. This reflects the effectiveness of the project in retaining cases and is comparable to other studies that showed high rates of successful outcomes of treated BU cases [24, 27]. The free treatment and socio-economic support provided by the project to the patients may have contributed to the success of the disease management. A previous study showed that even accessing highly-subsidised BU treatment programmes was challenging for patients, leading to many of them abandoning biomedical treatment altogether [28]. Future BU control projects in Nigeria will benefit from adopting the lessons learnt from the successes of this project.

A gap in our study is the lack of data on the HIV status of patients. In any case, there is little evidence from literature that there is an association between HIV and BU [29]. Secondly, unlike other studies in BU endemic settings [5-7], we adopted a strategy that combined different methods to detect the cases; a door-to-door survey may have yielded more cases.

\section{Conclusion}

In conclusion, this study has a number of strengths, including that it confirmed the endemicity of $\mathrm{BU}$ in the study population. We also identified-for the first time-more cases of clinical and laboratory-confirmed BU in Nigeria compared to any period in the past, as previous cases have not been sought. In addition, all treated cases completed chemotherapy. This study shows that BU disease is endemic in Ogoja territory of Cross River State and that the burden of the disease is likely to have been grossly underestimated in Nigeria. In all areas where BU has been reported in the last decade, the burden of the disease may be substantially higher than currently documented through the routine reporting system. We recommend that a phased systematic nationwide response based on lessons learnt from the pilot BU control programme be established-this may be integrated with National Tuberculosis and Leprosy Control Programme. We also believe that the identification and mapping of BU endemic regions in Nigeria should be strengthened.

\section{Additional file}

Additional file 1: Multilingual abstracts in the six official working languages of the United Nations. (PDF $329 \mathrm{~kb}$ )

\section{Abbreviations}

BU: buruli ulcer; DRC: Democratic Republic of Congo; LGA: local government area; qPCR: quantitative polymerase chain reaction; SBHO: St Benedict tuberculosis and leprosy rehabilitation hospital Ogoja; WHO: World Health Organization.

\section{Competing interests}

The authors declare that they have no competing interests.

\section{Authors' contributions}

$\mathrm{KNU}, \mathrm{AOM}, \mathrm{AC}, \mathrm{KBA}, \mathrm{JNC}, \mathrm{KLH}, \mathrm{ME}, \mathrm{MN}$ and $\mathrm{KN}$ participated in the conception and design of the study. KNU, AOM, JNC, MCA, CCN, DCO, NOM and NE analysed and interpreted the data. KNU, AOM, CCN, JNC, MCA, CCN, DCO, NOM and NE drafted the paper. All authors critically revised the paper for important intellectual content and approved it for submission.

\section{Acknowledgements}

The project was funded by Kindermissionswerk "Die Sternsinger" (Stephanstraße 35, D-52064 Aachen, Germany). Funding for laboratory testing, conducted at the Institute of Tropical Medicine Antwerp, was obtained from the Department of Economy, Science and Innovation of the Flemish Government. The funding bodies had no role in the study design; in the collection, analysis and interpretation of data; in the writing of the paper; and in the decision to submit the paper for publication. We also thank the WHO Geneva for its unwavering support, especially in providing the drugs and IEC materials for the project. We acknowledge the support of the Federal Ministry of Health, Nigeria through the NTBLCP. We thank the staff and management of the $\mathrm{SBHO}$ for their efforts and sustained commitment throughout the project. Finally, we acknowledge the support of all health workers, community leaders and patients for participating in the study.

\section{Author details}

'Department of Medicine, Federal Teaching Hospital, FMC Rd, Abakaliki, Ebonyi State, Nigeria. ${ }^{2}$ Medical Department, German Leprosy and TB Relief Association, Enugu, Enugu State, Nigeria. ${ }^{3}$ St Benedict's Tuberculosis and Leprosy Rehabilitation Hospital, Ogoja, Cross River State, Nigeria. ${ }^{4} G$ lobal Buruli Ulcer Initiative, Department of Control of Neglected Tropical Diseases, World Health Organization, Geneva, Switzerland. ${ }^{5}$ Department of Infectious Diseases and Tropical Medicine, University Hospital, Ludwig-Maximilians University, Munich, Germany. ${ }^{6}$ Mycobacteriology Unit, Department of Microbiology, Institute of Tropical Medicine, Antwerp, Belgium.

Received: 16 September 2015 Accepted: 14 March 2016 Published online: 22 April 2016

\section{References}

1. Huang GKL, Johnson PD. Epidemiology and management of Buruli ulcer. Expert Rev Anti Infect Ther. 2014;12:855-65.

2. World Health Organization. Buruli ulcer disease factsheet No 199, May 2015. http://www.who.int/mediacentre/factsheets/fs199/en/index.html (accessed July 9, 2015).

3. Debacker M, Aguiar J, Steunou C, Zinsou C, Meyers WM, Guedenon A, et al. Mycobacterium ulcerans disease (Buruli ulcer) in rural hospital, southern Benin, 1997-2001. Emerg Infect Dis. 2004;10:1391-8. 
4. Bratschi MW, Bolz M, Minyem JC, Grize L, Wantong FG, Kerber S, et al. Geographic distribution, age pattern and sites of lesions in a cohort of Buruli ulcer patients from the Mape' Basin of Cameroon. PLoS Negl Trop Dis. 2013;7:e2252.

5. Mavinga Phanzu D, Suykerbuyk P, Saunderson P, Ngwala Lukanu P, Masamba Minuku JB, Imposo DB, et al. Burden of Mycobacterium ulcerans disease (Buruli ulcer) and the underreporting ratio in the territory of Songololo, Democratic Republic of Congo. PLoS Negl Trop Dis. 2013;7:e2563.

6. Amofah G, Bonsu F, Tetteh C, Okrah J, Asamoa K, Asiedu K, et al. Buruli Ulcer in Ghana: results of a national case search. Emerg Infect Dis. 2002;8:167-70

7. Kanga JM, Kacou ED. Epidemiological aspects of Buruli ulcer in Cote d'Ivoire: results of a national survey. Bull Soc Pathol Exot. 2001;94:46-51.

8. Merritt RW, Walker ED, Small PLC, Wallace JR, Johnson PDR, Benbow ME, et al. Ecology and Transmission of Buruli Ulcer Disease: A Systematic Review. PLoS Negl Trop Dis. 2010;4:e911.

9. Johnson PD, Azuolas J, Lavender CJ, Wishart E, Stinear TP, Hayman JA, et al. Mycobacterium ulcerans in mosquitoes captured during outbreak of Buruli ulcer, southeastern Australia. Emerg Infect Dis. 2007;13:1653-60.

10. Gray HH, Kingma S, Kok SH. Mycobacterial skin ulcers in Nigeria. Trans R Soc Trop Med Hyg. 1967;61:712-4.

11. Oluwasanmi JO, Solankee TF, Olurin EO, Itayemi SO, Alabi GO, Lucas AO. Mycobacterium ulcerans (Buruli) skin ulceration in Nigeria. Am J Trop Med Hyg. 1976;25:122-8.

12. Chukwuekezie O, Ampadu E, Sopoh G, Dossou A, Tiendrebeogo A, Sadiq L, et al. Buruli ulcer, Nigeria. Emerg Infect Dis. 2007;13:782-3.

13. Vandelannoote K, Jordaens K, Bomans P, Leirs H, Durnez L, Affolabi D, et al. Insertion sequence element single nucleotide polymorphism typing provides insights into the population structure and evolution of Mycobacterium ulcerans across Africa. Appl Environ Microbiol. 2014;80:1197-209.

14. Noeske J, Kuaban C, Rondini S, Sorlin P, Ciaffi L, Mbuagbaw J, et al. Buruli ulcer disease in Cameroon rediscovered. Am J Trop Med Hyg. 2004;70:520-6.

15. Marion E, Carolan K, Adeye A, Kempf M, Chauty A, Marsollier L, et al. Buruli Ulcer in South Western Nigeria: a retrospective cohort study of patients treated in Benin. PLoS Negl Trop Dis. 2015;9:e3443.

16. World Health Organization. Incidence, prevalence and mapping of Buruli ulcer. http://www.who.int/buruli/research/priorities/healthmapping/ en/(accessed September 9, 2015)

17. The National Population Commission. 2006 Population and Housing Census of the Federal Republic of Nigeria. Priority tables (Volume I). Abuja: The National Population Commission; 2009.

18. World Health Organization. Laboratory diagnosis of buruli ulcer: a manual for health care providers. Portaels F, ed. WHO/HTM/NTD/IDM/2014.1, World Health Organization, 2014 Geneva

19. World Health Organization. Treatment of Mycobacterium ulcerans disease (Buruli ulcer): guidance for health workers, 2012. Available at: www.who.int/iris/bitstream/10665/77771/1/97892 41503402_eng.pdf (Accessed 10 July 2015).

20. Porten K, Sailor K, Comte E, Njikap A, Sobry A, Sihom F, et al. Prevalence of Buruli Ulcer in Akonolinga health district, Cameroon: results of a cross sectional survey. PLoS Negl Trop Dis. 2009;3:e466.

21. Johnson RC, Sopoh GE, Boko M, Zinsou C, Gbovi J, Makoutode M, et al. Distribution of Mycobacterium ulcerans Infection (Buruli Ulcer) in the District of Lalo in Benin. Trop Med Int Health. 2005;10:863-71.

22. Vincent QB, Ardant MF, Adeye A, Goundote A, Saint-André JP, Cottin J, et al. Clinical epidemiology of laboratory-confirmed Buruli ulcer in Benin: a cohort study. Lancet Glob Health. 2014;2:e422-30.

23. Barker DJP. Epidemiology of Mycobacterium ulcerans infection. Trans R Soc Trop Med Hyg. 1973;67:43-7.

24. Chauty A, Ardant MF, Adeye A, Euverte H, Guédénon A, Johnson C, et al. Promising clinical efficacy of streptomycin-rifampin combination for treatment of Buruli ulcer (Mycobacterium ulcerans disease). Antimicrob Agents Chemother. 2007:51:4029-35.

25. Mensah-Quainoo E, Yeboah-Manu D, Asebi C, Patafuor F, Ofori-Adjei D, Junghanss T, et al. Diagnostic of Mycobacterium ulcerans infection (Buruli ulcer) at a treatment centre in Ghana: a retrospective analysis of laboratory results of clinically diagnosed cases. Trop Med Int Health. 2008;13:191-8.
26. Stienstra Y, van der Werf TS, Guarner J, Raghunathan PL, Spotts Whitney EA, van der Graaf WT, et al. Analysis of an IS2404-based nested PCR for diagnosis of Buruli ulcer disease in regions of Ghana where the disease is endemic. J Clin Microbiol. 2003;41:794-97.

27. Kibadi K, Boelaert M, Fraga AG, Kayinua M, Longatto-Filho A, Minuku J-B, et al. Response to treatment in a prospective cohort of patients with large ulcerated lesions suspected to be Buruli Ulcer (Mycobacterium ulcerans Disease). PLoS Negl Trop Dis. 2010;4:e736.

28. Peeters Grietens K, Um Boock A, Peeters H, Hausmann-Muela S, Toomer E, Ribera JM. "It is me who endures but my family that suffers": social isolation as a consequence of the household cost burden of Buruli Ulcer free of charge hospital treatment. PloS Negl Trop Dis. 2008;2:e321.

29. Vincent QB, Ardant MF, Marsollier L, Chauty A, Alcaïs A, Franco-Beninese Buruli Research Group. HIV infection and Buruli ulcer in Africa. Lancet Infect Dis. 2014;14:796-7.

\section{Submit your next manuscript to BioMed Central and we will help you at every step:}

- We accept pre-submission inquiries

- Our selector tool helps you to find the most relevant journal

- We provide round the clock customer support

- Convenient online submission

- Thorough peer review

- Inclusion in PubMed and all major indexing services

- Maximum visibility for your research

Submit your manuscript at www.biomedcentral.com/submit
Biomed Central 